\title{
BMJ Open Substance use disorders among African, Caribbean and Black (ACB) people in Canada: a scoping review protocol
}

\author{
Joseph D Nguemo, ${ }^{1}$ Ngozi Iroanyah, ${ }^{2}$ Winston Husbands, ${ }^{3}$ LaRon E Nelson, ${ }^{4}$ \\ Geoffrey Maina, ${ }^{5}$ Irene Njoroge, ${ }^{6}$ Maureen Owino, ${ }^{7}$ Meldon Kahan, ${ }^{6}$ \\ Desmond Miller, ${ }^{8}$ Josephine Wong ${ }^{8}$
}

To cite: Nguemo JD, Iroanyah N, Husbands W, et al. Substance use disorders among African, Caribbean and Black (ACB) people in Canada: a scoping review protocol. BMJ Open 2019;9:e028985. doi:10.1136 bmjopen-2019-028985

- Prepublication history for this paper is available online. To view these files, please visit the journal online (http://dx.doi org/10.1136/bmjopen-2019028985).

Received 13 January 2019 Revised 22 May 2019 Accepted 7 June 2019

Check for updates

(c) Author(s) (or their employer(s)) 2019. Re-use permitted under CC BY-NC. No commercial re-use. See rights and permissions. Published by BMJ.

For numbered affiliations see end of article.

Correspondence to Dr Joseph D Nguemo; jnguemodjiometio@ryerson.ca

\section{ABSTRACT}

Introduction Previous research demonstrated that substance use continues to be one of the most complex and prevalent problems among African, Caribbean and Black (ACB) people. A number of studies were conducted to characterise substance use patterns in this population. To our knowledge, this is the first known review in Canada characterising substance use disorders on ACB people. This scoping review seeks to answer the following research questions: What characterises substance use disorders among ACB people in Canada? What are the different types and prevalence of substance use among ACB people in Canada? Do ACB people in Canada use more than one substance? What factors are associated with substance use among ACB people in Canada? What are the health and social impacts of substance use in ACB people in Canada?

Methods and analyses This study will use the methodological framework for scoping reviews developed by Arksey and 0'Malley. We will search electronic bibliographic databases including Ovid MEDLINE, PsycINFO and CINAHL. We will limit our search to English articles published between 2000and2019. In addition, we will conduct a grey literature search. Two investigators will independently screen citations and full-text articles. Our findings will be reported according to the Preferred Reporting Items for Systematic Reviews and MetaAnalysis for scoping reviews guidelines. We will provide a descriptive summary of the studies and summarise the findings with respect to the outcomes and report any gaps that might require further investigation.

Ethics and dissemination Our proposed study does not involve human participants; therefore, research ethics approval is not required. This study will provide evidence that will inform the development of strategies for appropriate interventions, as well as policy and further research. The results will be disseminated through publications in open access peer-reviewed journals, presentations at scientific meetings and to the lay public.

\section{BACKGROUND}

According to the 2016 Canadian census, 1198545 people self-identified as Black, representing $3.5 \%$ of Canada's total population and $16 \%$ of the total visible minority population. About $37 \%$ of the Black population in

\section{Strengths and limitations of this study}

- Our search strategy is very comprehensive, as it was developed with a team that has extensive experience with literature search and was reviewed in consultation with a medical research librarian.

- Our review uses a rigorous scoping review methodology and includes all types of study designs such as cohort, cross-sectional, systematic reviews, text and opinion.

- Our source of literature is expansive, as it includes three databases that cover our research questions as well as grey literature.

- This study is limited only to Canadian context and the findings will only be relevant to Canada.

- Another limitation of our study is the inclusion of literature published only in the English language.

Canada lives in Toronto. ${ }^{1}$ In Western countries, racialised minorities are more likely to face structural and social stressors that lead to health disparities and exacerbate poor health status. ${ }^{23}$ African, Caribbean and Black (ACB) people, especially those who are immigrants and refugees, experience a myriad of challenges and problems such as language barriers, exclusive immigration policies, unemployment, poverty, lack of access to healthcare, discrimination and racism. ${ }^{4}$ Research confirms that social inequities, systemic racial discrimination, sexism, poverty and marginalisation contribute to the compromised mental health among racialised people. ${ }^{5}$ Evidence also indicates that some marginalised ACB people turn to substance use as a coping strategy to overcome cumulative hardship and stressful life conditions. ${ }^{6}$ Depending on different factors such as the type of drugs used, doses, frequency and pre-existing health conditions, drug use can have various short-term and long-term health and social effects such as heart attack, crime, stroke, healthcare spending, drug dependence, change in blood pressure, violence, 
HIV/AIDS, hepatitis C virus, mental health issues, overdose and ultimately death. ${ }^{78}$

Like other Canadians, ACB people have a reported history of substance use. Data derived from multiple cycles (2005-2011) of the Canadian Community Health Survey-Mental Health in Ontario showed that $40 \%$ of Caribbean and $18 \%$ of African adults reported lifetime cannabis usage; also, 23\% of Caribbean and 9\% of African adults have reported using cannabis in the past year. ${ }^{9}$ The prevalence of problematic use of cannabis (that can lead to harm, abuse or dependence, moderate/high score of 8 or more) on the Alcohol, Smoking and Substance Involvement Screening Tool-Cannabis Involvement Score was $8 \%$ for Caribbean and $4 \%$ for African adults. ${ }^{9}$ In a community-based study of party drug use among ethno-racially diverse gay and bisexual men (Asian, Caribbean, Latino, Aboriginal, mixed and others) in Toronto, participants reported regular use of ecstasy, cannabis, ketamine and cocaine. ${ }^{10}$ In the same study, $43 \%$ of participants were polydrug users and almost all participants reported engaging in sex while they were on drugs. ${ }^{10}$ For women, data from the Canadian HIV Women's Sexual and Reproductive Health Cohort Study reported various types of drug users. Among participants who self-identified as ACB, 5\% used tobacco, $41 \%$ drank alcohol, $5 \%$ were 'socially acceptable' poly-substance users, $4 \%$ were illicit poly-substance users and 3\% were illicit poly-substance users of all types. ${ }^{11}$

Substance use is associated with increased risk of developing other mental disorders, utilisation of emergency rooms and can have severe impact not only on individuals, but also on those closest to them, and society in general. Population-based data from the Ontario HIV Treatment Network reported recreation drug use (RDU) (16\%) and co-occurring RDU and depression (34\%) among heterosexual individuals. Among specific ethnic groups (African, Caribbean, Asian or Latino), $14 \%$ reported RDU and 12\% reported co-occurring RDU and depression. ${ }^{12}$ Moreover, RDU-only or co-occurring depression and RDU were associated with increased incidence of emergency department (ED) visits. ${ }^{12}$ Previous observational study showed that opioid poisonings led to 16 hospitalisations per day in Canada. ${ }^{13}$ In the same study, it was found that substance use also increased ED visits. Over the last 5 years, heroin or synthetic opioid poisonings increased the number of ED visits by 10-fold in Alberta, whereas in Ontario, ED visits increased fourfold and doubled due to heroin and synthetic opioid poisonings, respectively. ${ }^{13}$ A study investigating substance use and intimate partner violence (IPV) showed that cocaine use was associated with IPV among Black men who have sex with men. ${ }^{14}$ Moreover, a significant association between illegal drug use and criminal behaviour exists. ${ }^{15}$ In several studies, it was demonstrated that alcohol can significantly increase the risk of sexual assault. ${ }^{16} \mathrm{~A}$ cross-sectional study investigating predictors of sexual assault found that the number of sexual assaults committed by African American and Caucasian men were associated with alcohol problems. ${ }^{17}$ Also, the relationship between driver blood alcohol concentration (BAC) and involvement in motor crash is well documented. Previous studies have reported that higher BACs significantly increase the likelihood of drivers to be involved in crashes. ${ }^{18}$ Substance use is associated with increased risky sexual behaviour and the risk of HIV acquisition. Evidence from a Canadian study showed that ACB women reported having sex under alcohol, substance use and drug influence; $2 \%$ had shared drug use equipment and $27 \%$ had ever mixed sex with drugs or alcohol and $44 \%$ of ACB men reported that they had engaged in sex while using drugs or alcohol. ${ }^{19}$

ACB in Canada experience different challenges that increase their vulnerability to use substances, however, the literature is rather sparse on this population. An initial search of the topic in PubMed, the JBI database of systematic reviews and implementation reports and PROSPERO did not identify previous reviews or any review currently underway. There is a need to characterise substance use disorders among this population. The proposed scoping review aims to explore the available research evidence regarding common substance use disorders by ACB people in Canada as well as their health and social impacts.

\section{METHODS AND ANALYSIS}

\section{Patient and public involvement}

Patients are not involved in the design of this scoping review study.

\section{Scoping review}

We will conduct a search of all research designs and types of publications, as well as grey literature and reports. The review will adhere to the Preferred Reporting Items for Systematic Reviews and Meta-Analysis Protocols (PRISMA-P) guidelines, including search strategy, selection criteria, data extraction and data analysis. ${ }^{20}$ Furthermore, the review will be conducted using the methodological framework for scoping reviews proposed by Arksey and O'Malley. ${ }^{21}$ The framework recommends the following six steps: (1) Identifying the research question, (2) identifying relevant studies, (3) study selection, (4) charting the data, (5) collating, summarising and reporting the results and (6) consultation. The review will be reported according to PRISMA for Scoping Reviews (PRISMA-ScR) guidelines and a checklist will be completed. ${ }^{22}$ Our scoping review was initiated on 1 December 2018 and is to be completed by 30 August 2019 .

\section{Step 1: Identifying the research question}

The following research questions of the scoping review were identified

1. What characterises substance use disorders among ACB people in Canada?

- What are the different types and prevalence of substance use among ACB people in Canada? 
Table 1 Database search strategy

\section{Ovid MEDLINE search}

\begin{tabular}{|c|c|c|}
\hline & Key term & \#Hits \\
\hline 1 & $\begin{array}{l}\text { exp ALCOHOLS/or Binge drink }{ }^{*} . \mathrm{mp} . \text { [mp=title, abstract, original title, } \\
\text { name of substance word, subject heading word, floating sub-heading } \\
\text { word, keyword heading word, protocol supplementary concept word, rare } \\
\text { disease supplementary concept word, unique identifier, synonyms] }\end{array}$ & 621613 \\
\hline 2 & (illicit adj2 drug*).tw. & 9827 \\
\hline 3 & Marijuana.mp. or exp Cannabis/ & 22250 \\
\hline 5 & Street Drugs/ & 10253 \\
\hline 6 & poppers.tw. & 338 \\
\hline 7 & exp COCAINE/or exp CRACK COCAINE/ & 24487 \\
\hline 8 & $\begin{array}{l}\text { (Amphetamines or N-Methyl-3,4- methylenedioxyamphetamineor 3,4- } \\
\text { Methylenedioxyamphetamine or Ecstasy or MDMA or MDA).tw. }\end{array}$ & 56092 \\
\hline 11 & Opiate*.tw. & 23681 \\
\hline 12 & exp OPIUM/ & 1961 \\
\hline 13 & exp BENZODIAZEPINES/ & 63290 \\
\hline 14 & Demerol.tw. or exp Meperidine/ & 5791 \\
\hline 15 & (Cigarette* or tabacco).tw. & 63819 \\
\hline 16 & $\begin{array}{l}\text { (Psychedelic adj2 drug*).mp. [mp=title, abstract, original title, name } \\
\text { of substance word, subject heading word, floating sub-heading word, } \\
\text { keyword heading word, protocol supplementary concept word, rare } \\
\text { disease supplementary concept word, unique identifier, synonyms] }\end{array}$ & 218 \\
\hline 17 & $\begin{array}{l}\text { (Hallucinogenic adj2 Drug*).mp. or hallucinogens.tw. [mp=title, abstract, } \\
\text { original title, name of substance word, subject heading word, floating } \\
\text { sub-heading word, keyword heading word, protocol supplementary } \\
\text { concept word, rare disease supplementary concept word, unique } \\
\text { identifier, synonyms] }\end{array}$ & 1630 \\
\hline 20 & exp FENTANYL/ & 15005 \\
\hline 21 & Sedative*.tw. & 17745 \\
\hline 22 & Valium.tw. or exp Diazepam/ & 17699 \\
\hline 23 & Xanax.tw. or Alprazolam/ & 1754 \\
\hline 24 & Ritalin.tw. or exp Methylphenidate/ & 6870 \\
\hline 25 & Adderall.tw. & 158 \\
\hline 26 & (Erectile adj2 dysfunction adj2 drug).tw. & 57 \\
\hline 27 & Viagra.tw. or Sildenafil Citrate/ & 5335 \\
\hline 28 & Cialis.tw. or Tadalafil/ & 1317 \\
\hline 29 & (Levitra or Vardenafil Dihydrochloride).tw. & 101 \\
\hline 30 & Crystal.tw. & 189761 \\
\hline 31 & (GHB or Gamma-hydroxybutyrate).tw. & 2544 \\
\hline 32 & canada/ & 85054 \\
\hline 33 & (Canada or Canad*).tw. & 112521 \\
\hline 34 & $\begin{array}{l}\text { (alberta or british columbia or nova scotia or prince edward island or } \\
\text { newfoundland or labrador or nunavut or northwest territories or Yukon or } \\
\text { Quebec or Saskatchewan or manitoba or Ontario or new brunswick).ti,ab. }\end{array}$ & 60865 \\
\hline
\end{tabular}


Table 1 Continued

\section{Ovid MEDLINE search}

\begin{tabular}{llr}
\hline & Key term & \#Hits \\
\hline 35 & Caribbean.mp. or exp Caribbean Region/ & 35105 \\
36 & $\begin{array}{l}\text { (Black* or Caribbean or African or Immigrant* or immigra* or migration or }^{*} \text { migrant }^{*} \text {.tw. }\end{array}$
\end{tabular}

\begin{tabular}{|c|c|c|}
\hline 37 & $\begin{array}{l}\text { (Black adj2 canadian*).mp. [mp=title, abstract, original title, name of } \\
\text { substance word, subject heading word, floating sub-heading word, } \\
\text { keyword heading word, protocol supplementary concept word, rare } \\
\text { disease supplementary concept word, unique identifier, synonyms] }\end{array}$ & 32 \\
\hline 39 & (new adj2 Canadian).tw. & 197 \\
\hline 40 & (asylum or new comer or refugees or minorit*).tw. & 66945 \\
\hline
\end{tabular}

42 substance-related disorders/or alcohol-related disorders/or amphetamine- 172286 related disorders/or cocaine-related disorders/ordrug overdose/or heroin dependence/orinhalant abuse/or marijuana abuse/oropioid-related disorders/or phencyclidine abuse/or psychoses, substance-induced/ or substance abuse, intravenous/orsubstance abuse, oral/orsubstance withdrawal syndrome/or "tobacco use disorder"/oropioid dependence. $\mathrm{mp}$.

\begin{tabular}{llr}
43 & Alcoholic Intoxication/ & 12144 \\
\hline 44 & exp OPIUM DEPENDENCE/ & 12 \\
45 & exp HEROIN DEPENDENCE/ & 250 \\
46 & substance addiction.tw. & 7594 \\
47 & (drug adj2 addiction).mp. [mp=title, abstract, original title, name of & \\
& substance word, subject heading word, floating sub-heading word, \\
& keyword heading word, protocol supplementary concept word, rare & disease supplementary concept word, unique identifier, synonyms]
\end{tabular}
word, subject heading word, floating sub-heading word, keyword heading word, protocol supplementary concept word, rare disease supplementary concept word, unique identifier, synonyms]

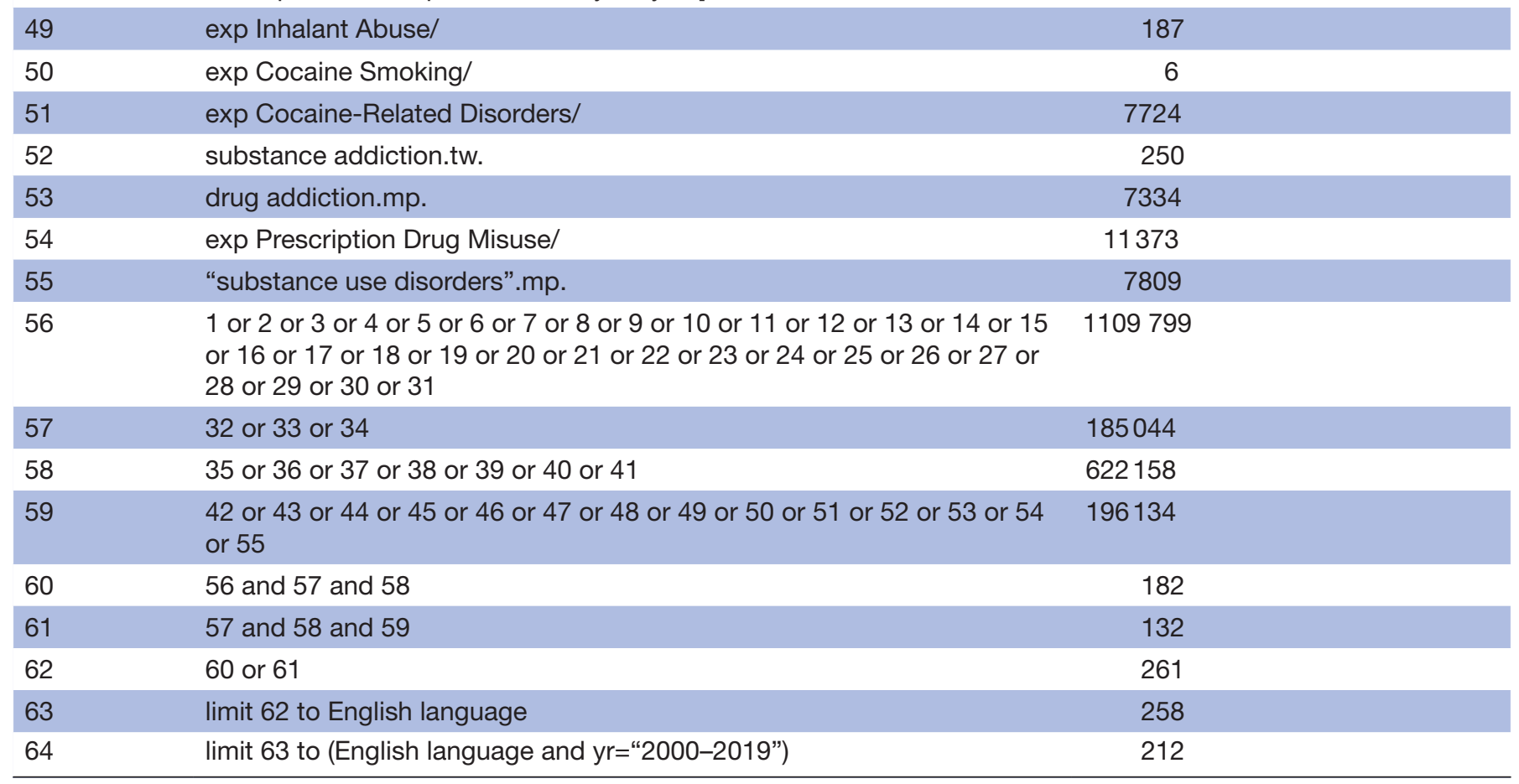


Table 1 Continued

\section{PsycINFO search strategy}

\section{Key terms}

(Black* OR Caribbean OR Africa* OR "Black Canadian" OR black-foreign OR africa-born OR foreign-born OR caribbean-born OR radicalized groups OR ethnic groups OR "Caribbean region" OR "African ancestry" OR "black Caribbean ethnicity") AND (canada OR ab(Canad* OR Canadian* OR alberta OR british columbia OR nova scotia OR prince edward island OR newfoundland OR labrador OR nunavut OR northwest territories OR Yukon OR Quebec OR Saskatchewan OR manitoba OR Ontario OR new brunswick)) AND (alcohol OR "illicit drug*" OR marijuana OR cannabis OR poppers OR "street drug" OR cocaine OR "stimulant drug*" OR amphetamines abuse OR n-methyl-3,4-methylenedioxyamphetamine OR 3,4-methylenedioxyamphetamine OR ecstasy OR hallucinogens OR methylenedioxymethamphetamine OR methylenedioxyamphetamine OR MDMA OR MDA OR psychedelic* OR psychotropic drug* OR heroin OR opiate* OR benzodiazepines OR demerol OR meperidine OR cigarette* OR tabacco OR "psychedelic drug*" OR "hallucinogenic drug*" OR "pain killer" OR vicodin OR codeine OR fentanyl OR sedative* OR valium OR diazepam OR xanax OR alprazolam OR ritalin OR methylphenidate OR party drug* OR adderall OR "erectile dysfunction drug" OR viagra OR sildenafil citrate OR cialis OR tadalafil OR levitra OR "poly drug" OR crystal OR gammahydroxybutyrate OR gamma-hydroxybutyrate OR vardenafil OR "recreation drug*" or (substancerelated disorders) OR (alcohol-related disorders) OR (amphetamine-related disorders) OR (cocaine-related disorders) OR (drug overdose) OR (heroin dependence) OR (inhalant abuse) OR (marijuana abuse) OR (opioid-related disorders) OR (phencyclidine abuse) OR psychoses OR substance-induced OR (substance abuse) OR intravenous OR (drug injection) OR (substance withdrawal syndrome) OR (tobacco use disorder) OR (opioid dependence) OR (Alcoholic Intoxication) OR (opium dependence) OR (heroin dependence) OR (substance addiction) OR (drug abuse) OR (Inhalant Abuse) OR (Cocaine Smoking) OR (Cocaine-Related Disorders) OR (substance addiction) OR (drug addiction) OR "Prescription Drug Misuse" OR "substance use disorders") Limits: English, 2000-01-01 - 2019-04-05.

\section{\#Hits}

321

\section{CINHAL search strategy}

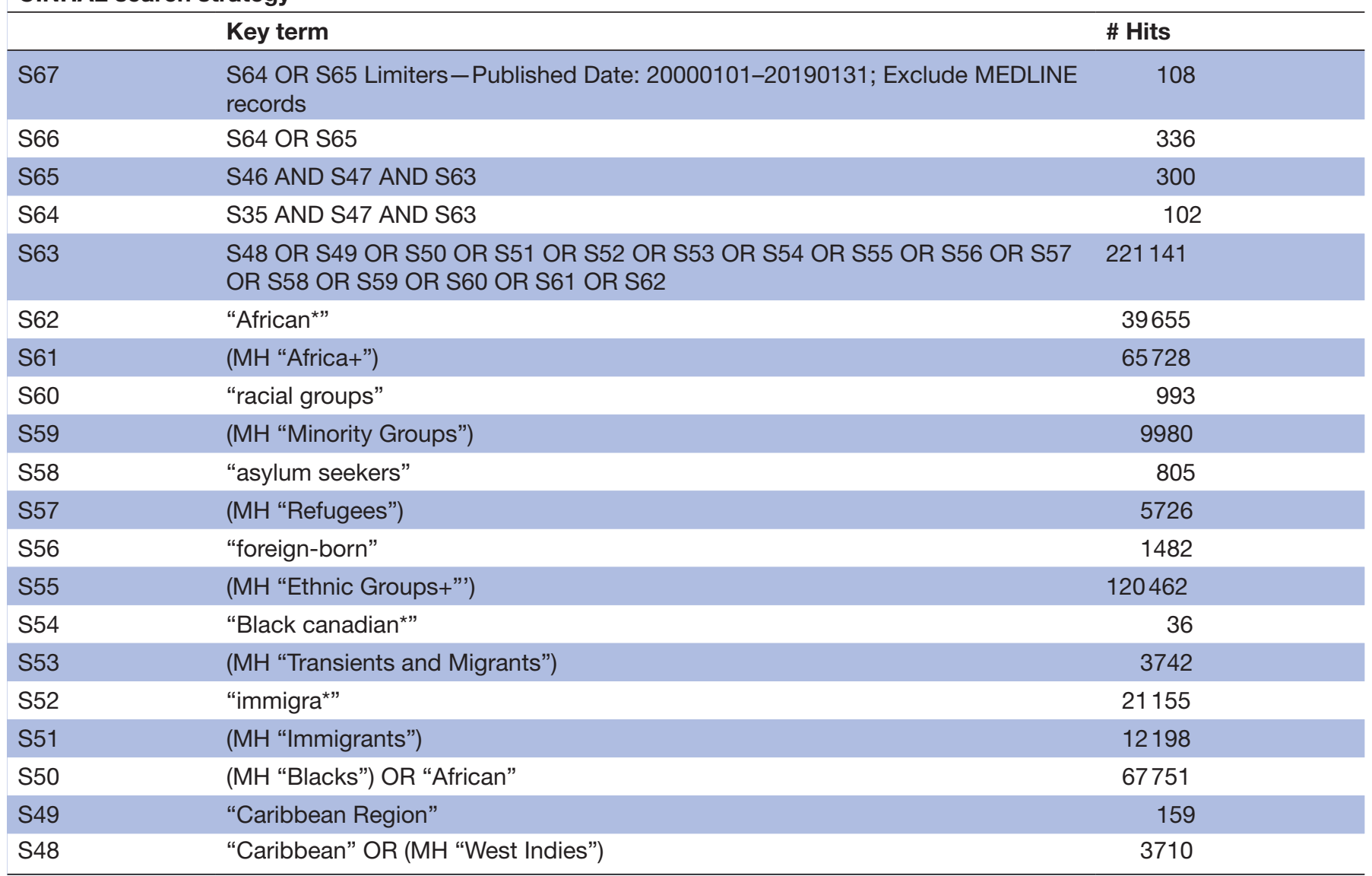

Continued 
Table 1 Continued

\section{CINHAL search strategy}

\begin{tabular}{|c|c|c|}
\hline & Key term & \# Hits \\
\hline S47 & $\begin{array}{l}\text { (MH “Canada") OR (MH "Alberta") OR (MH "British Columbia”) OR (MH } \\
\text { "Manitoba") OR (MH "New Brunswick") OR (MH "Newfoundland") OR (MH } \\
\text { "Northwest Territories") OR (MH "Nova Scotia") OR (MH "Nunavut") OR (MH } \\
\text { "Ontario") OR (MH "Quebec") OR (MH "Saskatchewan") OR (MH "Yukon } \\
\text { Territory") }\end{array}$ & 87270 \\
\hline S46 & S36 OR S37 OR S38 OR S39 OR S40 OR S41 OR S42 OR S43 OR S44 OR S45 & 149256 \\
\hline S44 & (MH “Inhalant Abuse”) & 340 \\
\hline S43 & "PRESCRIPTION DRUG MISUSE" & 279 \\
\hline S42 & (MH “Intravenous Drug Users”) & 1787 \\
\hline S41 & (MH "Substance Abuse+") & 56700 \\
\hline S40 & MH “Alcohol-Induced Disorders" & 670 \\
\hline S37 & "drug addiction" & 2243 \\
\hline S36 & $\begin{array}{l}\text { (MH "Substance Use Disorders+") OR "substance addiction" OR (MH "Behavior, } \\
\text { Addictive+") OR (MH "Substance Use Rehabilitation Programs+") OR (MH } \\
\text { "Substance Dependence+") }\end{array}$ & 145870 \\
\hline S35 & 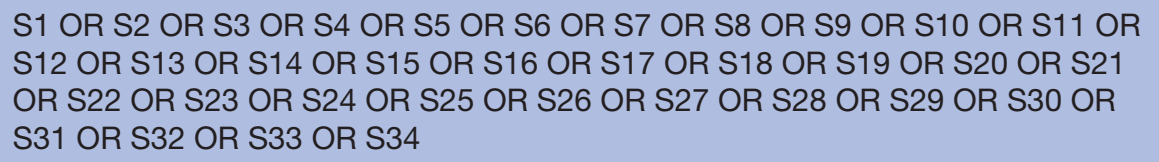 & 151279 \\
\hline S34 & (MH “Analgesics, Opioid+”) & 29420 \\
\hline S33 & "recreational drugs" & 238 \\
\hline S26 & "Erectile dysfunction drug" & 95 \\
\hline S25 & “Adderall" & 92 \\
\hline S24 & (MH “Methylphenidate") OR "Ritalin" & 2132 \\
\hline S23 & (MH "Methylphenidate") & 2062 \\
\hline S22 & (MH “Alprazolam”) OR “Xanax” & 332 \\
\hline S21 & (MH “Diazepam”) & 972 \\
\hline S20 & "Sedative*" & 8160 \\
\hline S19 & (MH “Fentanyl+") & 4125 \\
\hline S18 & (MH “Codeine+”) OR (MH “Oxycodone') & 2217 \\
\hline S17 & "Vicodin" & 37 \\
\hline S16 & "Hallucinogenic Drug"” & 40 \\
\hline S15 & (MH “Hallucinogens+") & 1587 \\
\hline S14 & $\begin{array}{l}\text { (MH “Street Drugs+"') OR (MH “Drugs, Non-Prescription”) OR “Psychedelic } \\
\text { drug"”" }\end{array}$ & 9003 \\
\hline S13 & (MH “Meperidine") OR “Demerol” & 764 \\
\hline S12 & (MH “Antianxiety Agents, Benzodiazepine+”) & 8068 \\
\hline
\end{tabular}




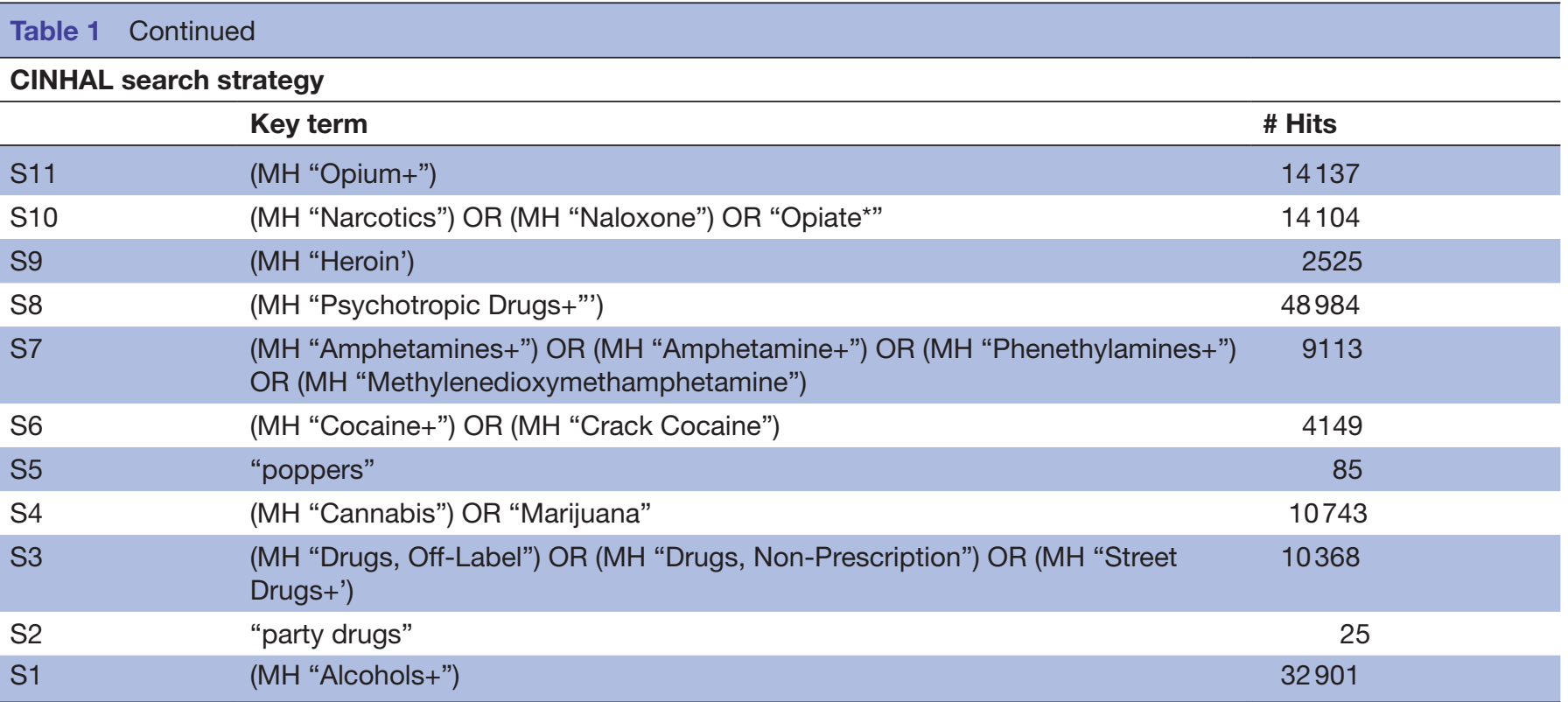

- Do ACB people in Canada use more than one substance?

- What are the factors associated with substance use among ACB people in Canada?

2. What are the health and social impacts of substance use among ACB people in Canada?

The following population, intervention, comparison, outcome was formulated:

1. Population: Canadian ACB people.

2. Intervention: Study examines substance use.

3. Comparison: Other ethnic groups if provided.

4. Outcomes:

- Types of substance use disorders.

- Prevalence of substance use disorders and poly drugs use.

- Health impact of substance use.

- Social impact of substance use.

\begin{tabular}{lll}
\hline Table 2 & Web-based search strategy: Google search engine \\
\hline No. & Search term & \# of Hits \\
\hline 1 & $\begin{array}{l}\text { Substance abuse or } \\
\text { substance use or or name } \\
\text { of substance, eg, alcohol, } \\
\text { cannabis, poppers }\end{array}$ & \\
& $\begin{array}{l}\text { Blacks or Caribbean or } \\
\text { African }\end{array}$ & TBD \\
3 & $\begin{array}{l}\text { Canada } \\
\text { \#1 and \#2 and \#3 }\end{array}$ & TBD \\
& $\begin{array}{l}\text { First five-pages of the } \\
\text { web-based search will be } \\
\text { reviewed } \\
\text { Limitation: }\end{array}$ \\
& $\begin{array}{l}\text { 1. English. } \\
\text { 2. 2000-present. }\end{array}$ \\
\hline
\end{tabular}

TBD, to be decided.
- Factors associated with substance use.

\section{Step 2: Identifying relevant studies}

In order to address the objectives of the review, an internet-based search of published and grey literature will be conducted using electronic databases and Google. Also, we will search the website of key organisations, such as Health Canada, Statistics Canada and the Canadian Centre on Substance Use and Addiction. For each individual database, our search strategy will combine Medical Subject Headings and 'free-text' terms in conjunction with the Boolean operators 'and' and 'or'. We will conduct searches in relevant electronic databases: Ovid MEDLINE, PsycINFO and CINHAL.

Our search will be limited to English language publications. Only studies published between 2000 and 2019 will be included in this review. Our search strategy is described in tables 1-3. Also, we will check reference lists of included studies to identify any additional relevant articles that were not captured by our search strategy.

Table 3 Health Canada, Statistic Canada and the Canadian Centre on substance use and addiction search strategy

\begin{tabular}{lll}
\hline No. & Search term & \# of hits \\
\hline 1 & $\begin{array}{l}\text { Substance abuse or substance use } \\
\text { or name of substance, eg, alcohol, } \\
\text { cannabis, poppers, etc. }\end{array}$ & TBD \\
& $\begin{array}{l}\text { And } \\
\text { African or Caribbean or Blacks }\end{array}$ \\
& Limitation: & TBD \\
& 1. English & \\
2. 2000-present &
\end{tabular}

TBD, to be decided. 


\section{Box 1 Inclusion criteria}

\section{Eligibility checklist}

Inclusion criteria

Population: Canadian ACB male and female.

Intervention: Study examines substance use.

Comparison: Other ethnic groups if provided.

Outcomes: at least one of the following:

- Types of substance use disorders.

- Prevalence of substance use or poly drugs use.

- Factors associated with substance use.

- Health impact of substance use.

- Social impact of substance use.

Exclusion criteria

Article describes normal prescription drug use.

\section{Step 3: Study selection}

We will use Endnote 9 (Clarivate Analytics), a citation management software programme, to manage references and remove duplicates. To facilitate study screening and selection, all citations obtained using the search strategy will be imported into Rayyan, a web and mobile application for systematic reviews. ${ }^{23}$ Study selection will proceed according to the stages described below.

\section{Stage 1}

A customised form reflecting the inclusion criteria in box 1 will be pilot tested by two reviewers. Specifically, a screening form will be developed and will be applied by two reviewers independently to a sample of $5 \%$ abstracts to ensure consistency of use and clarity of the instrument. A Cohen's kappa statistic ${ }^{24}$ will be estimated to measure inter-rater reliability, and screening will begin when $>60 \%$ agreement is achieved.

\section{Stage 2: Assessment of studies for inclusion \\ Inclusion criteria}

To be included in this study a reference should meet the following criteria:

1. Population: Canadian ACB people.

2. Intervention: Study examines substance use.

3. Comparison: Other ethnic groups if provided.

4. Outcomes-Study examines at least one of the following:

- Types of substance use disorders.

- Prevalence of substance use disorders and poly drugs use.

- Health impact of substance use.

- Social impact of substance use.

- Factors associated with substance use.

All titles and abstracts identified from the electronic database search will be reviewed independently by two reviewers. Differences in opinion will be resolved by consensus. Discussion with a third author will take place in situations where consensus cannot be reached. In cases where abstracts are not provided, are unclear, or there is any other reason for uncertainty, the full article will be obtained before making a decision regarding eligibility

\section{Box 2 Data extraction form}

Study authorStudy author

TitleTitle

YearYear

ProvinceProvince

PurposePurpose

DesignDesign

MethodMethod

Sample sizeSample size

Population gender (M/F)Population gender (M/F)

Comparator if applicableComparator if applicable

Age (youth: 16-25year; adult >25year)Age (youth: 16-25year; adult $>25$ year)

OutcomesOutcomes

- Type of substance use disorders (stimulant, depressant, hallucinogen, opioids, inhalants, cannabis, alcohol, tobacco).

- Prevalence of substance use and poly drugs use.

- Associated factors of substance use.

- Social impact.

- Health impact.

Main findingsMain findings

LimitationsLimitations

ConclusionConclusion

for inclusion. The full text of all potentially relevant articles will be obtained and will be reviewed using the inclusion criteria defined above.

\section{Exclusion criteria}

Articles that describe normal prescription drug use.

\section{Step 4: Charting the data}

Eligible studies will be extracted using a pre-designed data extraction form. The data extraction form will be pilot tested with a sample of five studies to ensure clarity and consistency. We will extract basic study information like first author, title, purpose, year of publication and province. In addition, we will extract information about the population, method, age, type of substance use, poly drug use, impact of drug use, findings and limitations of the study (box 2).

\section{Step 5: Collating, summarising and reporting the results}

Rather than provide a quantitative synthesis of literature, as is typically the use of systematic reviews, this scoping review aims to summarise a wide range of findings regarding substance use disorders among ACB people in Canada. ${ }^{25}$ Therefore, we will provide a descriptive summary of the gathered articles including peer-reviewed articles, text, opinion or systematic reviews. The descriptive summary will contain the characteristics of included studies, such as the overall number of studies, types of study design, years of publication, characteristics of the study populations and provinces where studies were conducted. In addition, we will summarise the study findings with respect to the outcomes and report any gaps that might require further investigation. Our synthesis will inform whether ACB people in Canada experience disorders related to alcohol, tobacco, stimulants, 
depressants, hallucinogens, opioids or cannabis. Also, our synthesis will inform whether ACB people in Canada use more than one drug at a time and, the health and social impacts of substance use.

\section{Step 6: Consultation}

Neither patients nor public will be involved.

\section{ETHICS AND DISSEMINATION}

Our proposed study does not involve human participation. As a scoping review, this study will use only published literature. Therefore, research ethics approval is not required. Results will be disseminated through publications in open access peer-reviewed scientific journals, presentations at scientific meetings and presentations to the lay public through the media where appropriate. The results from this review will be used to plan future systematic reviews.

\section{DISCUSSION AND CONCLUSION}

The proposed scoping review will have the potential to inform research, programmes and services that could be used to improve the health and well-being of ACB people in Canada. Specifically, this review will inform policy-makers, healthcare providers, clinicians and researchers on substance use among Canadian ACB people.

\section{Author affiliations}

${ }^{1}$ Daphne Cockwell School of Nursing, Ryerson University, Toronto, Ontario, Canada ${ }^{2}$ School of health policy and management, York University, Toronto, Ontario, Canada ${ }^{3}$ Ontario HIV Treatment Network, Toronto, Ontario, Canada

${ }^{4}$ Li Ka Shing Knowledge Institute, Unity Health Toronto, Toronto, Ontario, Canada

${ }^{5}$ College of Nursing, University of Saskatchewan, Saskatoon, Saskatchewan, Canada

${ }^{6}$ Rapid Access Addiction Medicine Clinic, Women's College Hospital, Toronto, Ontario, Canada

${ }^{7}$ Committee for Accessible AIDS Treatment, Regent Park Community Health Centre, Toronto, Ontario, Canada

${ }^{8}$ Daphne Cockwell School of Nursing, Ryerson University, Toronto, Ontario, Canada

Acknowledgements We thank the medical librarian at Ryerson University, Don Kinder, for advising our team on search strategies and available resources.

Contributors Conception of the study: JDN, WH, LEN, JW. Revision of the research questions: JDN, WH, JW. Drafting the manuscript: JDN, NI. Developing search strategy: JDN, DM. Revising the manuscript critically for important intellectual content: JDN, DM, LEN, WH, JW. Approval of the final version of the manuscript: JDN, NI, WH, LEN, GM, NI, OM, KM, DM, JW. Guarantor of the review: JDN.

Funding Joseph Nguemo Djiometio is a Posdoctoral fellow at Ryerson University; he is supported by CIHR/OHTN through the weSpeak project. The paper was also made possible through the support of the OHTN Applied HIV Research Chair in HIV Program Science with African, Caribbean and Black Communities \#AHRC-1066.

Competing interests None declared.

Patient consent for publication Not required.

Provenance and peer review Not commissioned; externally peer reviewed.

Open access This is an open access article distributed in accordance with the Creative Commons Attribution Non Commercial (CC BY-NC 4.0) license, which permits others to distribute, remix, adapt, build upon this work non-commercially, and license their derivative works on different terms, provided the original work is properly cited, appropriate credit is given, any changes made indicated, and the use is non-commercial. See: http://creativecommons.org/licenses/by-nc/4.0/.

\section{REFERENCES}

1. Canada S. Diversity of the Black population in Canada: An overview. Ottawa: Statistics Canada, 2019. Retrieved from. https://www150. statcan.gc.ca/n1/en/pub/89-657-x/89-657-x2019002-eng.pdf?st= IM3POXWu.

2. Chung B, Meldrum M, Jones F, et al. Perceived sources of stress and resilience in men in an African American community. Prog Community Health Partnersh 2014;8:441-51.

3. Spence ND, Wells S, Graham K, et al. Racial Discrimination, Cultura Resilience, and Stress. Can J Psychiatry 2016;61:298-307.

4. Assari S, Caldwell CH. Social Determinants of Perceived Discrimination among Black Youth: Intersection of Ethnicity and Gender. Children 2018;5:24.

5. Mays VM, Jones AL, Delany-Brumsey A, et al. Perceived Discrimination in Health Care and Mental Health/Substance Abuse Treatment Among Blacks, Latinos, and Whites. Med Care 2017;55:173-81.

6. Stevens-Watkins D, Perry B, Harp KL, et al. Racism and Illicit Drug Use Among African American Women: The Protective Effects of Ethnic Identity, Affirmation, and Behavior. J Black Psychol 2012;38:471-96.

7. Lafaye G, Karila L, Blecha L, et al. Cannabis, cannabinoids, and health. Dialogues Clin Neurosci 2017;19:309-16.

8. Des Jarlais DC, Cooper HLF, Arasteh K, et al. Potential geographic "hotspots" for drug-injection related transmission of HIV and HCV and for initiation into injecting drug use in New York City, 2011-2015, with implications for the current opioid epidemic in the US. PLOS One 2018;13:e0194799.

9. Tuck A, Hamilton HA, Agic B, et al. Past year cannabis use and problematic cannabis use among adults by ethnicity in Ontario. Drug Alcohol Depend 2017;179:93-9.

10. Greenspan NR, Aguinaldo JP, Husbands W, et al. "It's not rocket science, what I do": Self-directed harm reduction strategies among drug using ethno-racially diverse gay and bisexual men. Int $J$ Drug Policy 2011;22:56-62.

11. Carter A, Roth EA, Ding E, et al. Substance Use, Violence, and Antiretroviral Adherence: A Latent Class Analysis of Women Living with HIV in Canada. AIDS Behav 2018;22:971-85.

12. Choi SKY, Boyle E, Cairney J, et al. Impact of depression and recreational drug use on emergency department encounters and hospital admissions among people living with HIV in Ontario: A secondary analysis using the OHTN cohort study. PLoS One 2018;13:e0195185.

13. Grywacheski V, O'Connor S, Louie K. Opioid-Related Harms in Canada. Healthc Q 2018;20:10-12.

14. Wu E, El-Bassel N, McVinney LD, et al. The association between substance use and intimate partner violence within Black male samesex relationships. J Interpers Violence 2015;30:762-81.

15. Yitayih Y, Abera M, Tesfaye E, et al. Substance use disorder and associated factors among prisoners in a correctional institution in Jimma, Southwest Ethiopia: a cross-sectional study. BMC Psychiatry 2018:18:314.

16. Abbey A. Alcohol's role in sexual violence perpetration: theoretical explanations, existing evidence and future directions. Drug Alcohol Rev 2011;30:481-9.

17. Abbey A, Parkhill MR, BeShears R, et al. Cross-Sectional Predictors of Sexual Assault Perpetration in a Community Sample of Single African American and Caucasian Men. Aggress Behav 2006;32:54-67.

18. Yao J, Voas RB, Lacey JH. Drivers with alcohol use disorders and their risks of crash involvement. Drug Alcohol Depend 2018;183:210-6.

19. Baidoobonso S, Bauer GR, Speechley KN, et al. HIV risk perception and distribution of HIV risk among African, Caribbean and other Black people in a Canadian city: mixed methods results from the BLACCH study. BMC Public Health 2013;13:184.

20. Moher D, Shamseer L, Clarke M, et al. Preferred reporting items for systematic review and meta-analysis protocols (PRISMA-P) 2015 statement. Syst Rev 2015;4:1.

21. Arksey H, O'Malley L. Scoping studies: towards a methodological framework. Int J Soc Res Methodol 2005;8:19-32.

22. Tricco AC, Lillie E, Zarin W, et al. PRISMA Extension for Scoping Reviews (PRISMA-ScR): Checklist and Explanation. Ann Intern Med 2018;169:467-73.

23. Ouzzani M, Hammady H, Fedorowicz Z, et al. Rayyan-a web and mobile app for systematic reviews. Syst Rev 2016;5:210.

24. Viera AJ, Garrett JM. Understanding interobserver agreement: the kappa statistic. Fam Med 2005;37:4.

25. Levac D, Colquhoun H, O'Brien KK. Scoping studies: advancing the methodology. Implement Sci 2010;5:69. 